\title{
Identification of RBC and WBC Count in Human Blood Using ARM Based Instrumentation
}

\author{
B.Dodda Basavanagoud \\ Dept. of Electronics \& Communication Engineering \\ R.Y.M Engineering College, \\ Bellary, Karnataka, India, \\ Email:dbg_rymec@rediffmail.com
}

\author{
Dr. K Padma Priya \\ Dept. of Electronics \& Communication Engineering \\ JNTUK College of Engineering \\ Vizianagaram, Andhrapradesh, India
}

\begin{abstract}
The rapid growth in microelectronics and crunching RISC in the field of bio-medical sciences incorporated of soft tools to diagnose various parameters of human fluids. Conventional method of blood sample analysis makes use of laboratory technique of titration, which is operator-dependent and results in lot of errors depending on the skill of the technician. In order to eliminate the human errors involved in the conventional method, in this paper an attempt has been made to present a capillary centrifuge technique driven by high speed DC motor fed by Morgan chopper and controlled by powerful ARM processor. It results in accurate analysis of the blood samples. The various techniques involved in accurate sensing of speed using timer and generation of firing pulses to thyristor in the Morgan chopper is judiciously achieved. This paper clearly brings out the advantages of the proposed blood measurement technique which effectively gives blood analysis faster and at a low cost.
\end{abstract}

Index Terms- Capillary tube; DC Motor; Optical Encoder; Step-up Morgan chopper; ARM controller, RBC (red blood corpuscles), WBC (white blood corpuscles).

\section{INTRODUCTION}

Evolutions in modern electronics have led to a new beginning in the field of bio-medical sciences. As a result, various sophisticated equipments have been developed in the recent past. These instruments have helped the physicians to treat the patients effectively. Further many instruments related to blood analysis have also been developed, but these equipments are too expensive and beyond the reach of medium scale laboratories and clinics. Hence, many attempts to develop a cost effective method or technique is needed. One such attempt to develop a simple instrument and technique to measure blood sample is presented in this paper.

In this paper, a DC motor driven by chopper holds a capillary with blood sample and is rotated at high speed on time reference to give RBC and WBC count data. An attempt is made to design a low cost blood analyzer incorporating centrifuge technique. This method is simple and straightforward and it yields quick results. The centrifuge is effectively controlled by an ARM Controller and a chopper circuit to run the motor at $10,000 \mathrm{rpm}$. It also measures the blood information and displays it. The data obtained is compared with the result of the standard equipment to ensure good results [3-18].

\section{Optimization OF CAPILlary TUBE}

A capillary tube is a long, narrow tube of a fixed diameter. Typical tube diameters of capillary tubes range from $2 \mathrm{~mm}$ to $6 \mathrm{~mm}$ and vary in length. The capillary tube selected for experimentation has the diameters of $2 \mathrm{~mm}$ and length $10 \mathrm{~cm}$. The choice of the capillary tube is based on the series of experimentation carried out on different capillary tubes. Based on the above process, the capillary tube is optimized. The readings of capillary rotated for different time periods is shown in fig. 1 and is plotted with time reference that yields blood dissociation. The graph clearly indicates that the optimized capillary tube gives effective results when rotated at $10000 \mathrm{rpm}$ for $5 \mathrm{~min}$. The results are tabulated in table.1 to obtain the panoramic view of the capillary tubes and its response for various speeds in conjunction with the blood layers formed.

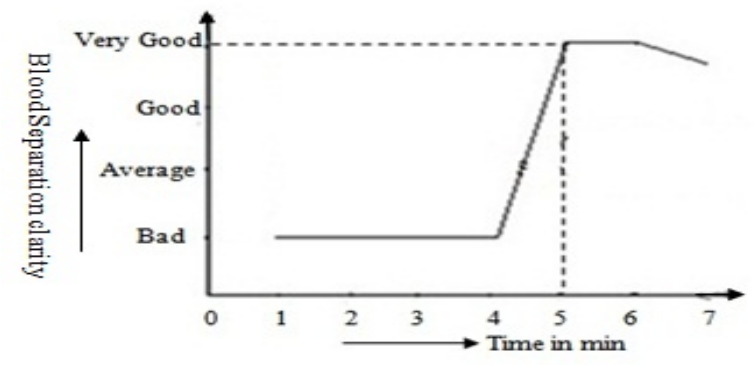

Fig.1. Optimized Graph of Capillary tube

TABLE I. OPTIMIZATION OF CAPILLARY TUBE

\begin{tabular}{|c|c|c|c|c|}
\hline \multirow{2}{*}{$\begin{array}{c}\text { Sl. } \\
\text { No. }\end{array}$} & $\begin{array}{c}\text { Diameter } \\
\text { (mm) of } \\
\text { capillary } \\
\text { tube }\end{array}$ & $\begin{array}{c}\text { DC Motor } \\
\text { Speed (rpm) }\end{array}$ & $\begin{array}{c}\text { Spinning } \\
\text { time } \\
\text { (min) }\end{array}$ & $\begin{array}{c}\text { Observation of } \\
\text { blood sample in } \\
\text { capillary }\end{array}$ \\
\hline \multirow{4}{*}{1} & 2 & $1000-10000$ & 3 & No layers formed \\
\cline { 2 - 5 } & 2 & $1000-10000$ & 4 & No layers formed \\
\cline { 2 - 5 } & 2 & $1000-6000$ & 5 & No layers formed \\
\cline { 2 - 5 } & 2 & $6001-9000$ & 5 & $\begin{array}{c}\text { Interleaving of RBC, } \\
\text { WBC \& plasma }\end{array}$ \\
\hline
\end{tabular}




\begin{tabular}{|c|c|c|c|c|}
\hline & 2 & 10000 & 5 & $\begin{array}{c}\text { Three distinct layers } \\
\text { formed }\end{array}$ \\
\hline \multirow{3}{*}{2} & 3 & $1000-10000$ & 3 & No layers formed \\
\cline { 2 - 5 } & 3 & $1000-10000$ & 4 & No layers formed \\
\cline { 2 - 5 } & 3 & 7000 & 5 & $\begin{array}{c}\text { Interleaving of WBC } \\
\text { and plasma }\end{array}$ \\
\hline 3 & 4 & $1000-10000$ & $3-5$ & No layers formed \\
\hline 4 & 6 & $1000-10000$ & $3-5$ & No layers formed \\
\hline
\end{tabular}

III. FUNCTIONAL DIAGRAM

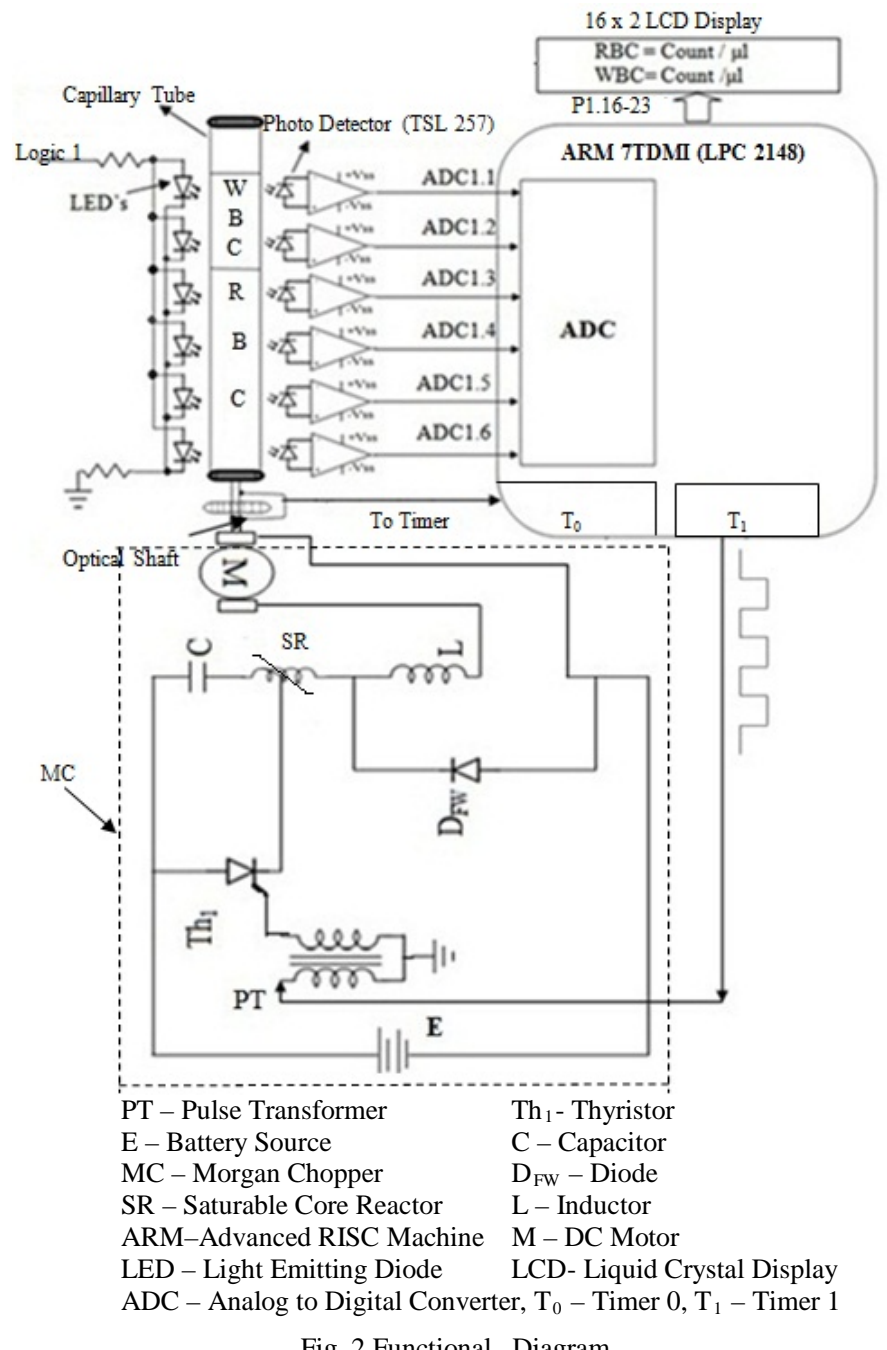

Fig. 2.Functional Diagram

Functional diagram of the system for separation and detection of the whole blood components is shown in the fig.2.

\section{A. Motor Drive}

In order to separate the different components, present in a whole blood sample, such as RBC, WBC, Platelets \& Plasma, a 12 V, 3.3 Amp, 12000 rpm, DC motor driven by chopper is used in centrifugal system, which has to rotate at a speed of $10000 \mathrm{rpm}$ for a duration of $5 \mathrm{~min}$. Speed of DC motor is controlled by using Morgan chopper.

\section{B. Photo Detector}

The photo detector used in the system is in the form of a single monolithic integrated circuit of TSL 257. It is a high sensitivity, low noise and light to voltage optical converter. It consists of a photo diode and transimpedence amplifier with a transimpedence gain of $\mathrm{M} \Omega$. It comes in the form of three lead plastic packages with an integral lens [21], improved offset voltage stability and it consumes less power. Light intensity to voltage detector and LED light sources are placed opposite to each other in the form of columns as shown in fig.2 on either side of the sample holder. The ARM processor gets input from the detectors using on-chip A/D converter. The Key features of photo detector are Converting light intensity into voltage, IC containing photodiode and op-amp, High sensitivity to single voltage supply operation, Low noise rail-to-rail output and High power supply rejection ratio.

\section{ARM LPC 2148}

The Arm controller has built in ADC(s) of one having 6 analog input channels and the other having 4 analog input channels. Both the ADC(s) have 10 bit digital outputs. The present paper aims at the data acquisition function that enables reading of the voltages available from the photo detectors that are fed to the ADC having 6 channels [22,23,24]. An ALP is written to acquire this data for further processing. The speed is measured by taking into account the pulses obtained by the optical shaft and feeding it to the timer (0).The principle of speed measurement is explained in the preceding section.

\section{Speed Measurment \& Generation of Triggering Pulses to SCR.}

A program has been developed to sense the zero instant of the square wave obtained from the optical shaft. The ARM measures the magnitude of square wave at two consecutive points as shown in fig.3. The two magnitudes are compared and a conclusion is derived on the basis of carry and zero status flags, irrespective of the point being at zero instant. Various points have been shown in fig.3. Very near to P3 at its left side the magnitude of square wave is zero and at $\mathrm{P} 45 \mathrm{~V}$ at logic ' 1 '. The ARM subtracts the 1st value from the 2 nd, so the result is non zero and there is no carry. This is the basis for the selection of zero instant point. Suppose reading is taken at P1 \& P2 where both magnitudes are zero, difference of the two is zero. So, this is not the zero instant of the wave at points P5 \& P6. The difference of the two values is zero, so it is also not a zero instant point at P7 \& P8. The difference is non zero but there is a carry. So it is the end point of the half-square wave. As soon as the zero instant point is detected, the ARM initiates a register to count the number of times the loop is executed. The ARM reads the magnitude of the square wave again and again \& moves in the loop. It crosses the loop when the magnitude of the square wave becomes zero. Thus the time for half cycle is measured. The count can be compared with the stored numbers in a look up table and the speed can be displayed. 


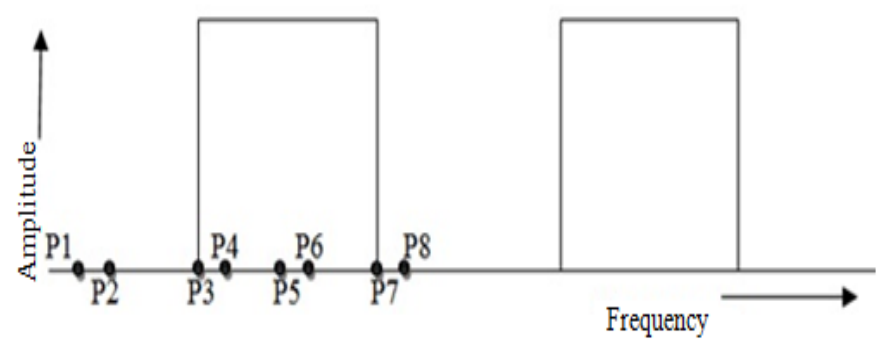

Fig.3 Square Wave of Speed Measurement

Once the speed $\mathrm{N}$ of the motor is evaluated, it is compared with the set speed of $10000 \mathrm{rpm}$. If the speed is less than the set i.e $10000 \mathrm{rpm}$, a new firing pulse is generated by picking up a new firing angle $\alpha$ from the look up table.

\section{DC MOTOR \& MORGAN CHOPPER}

The DC machine is a highly versatile machine. It can provide high starting torques as well as high accelerating and rotating torques. It is capable of quick speed reversals and it controls speed at a range of $4: 1$, with a relative ease in comparison with all other electromechanical energy-conversion devices.

The uniqueness of this circuit is that a saturable core reactor (SR) replaces the inductor for the purpose of LC oscillation. The load is assumed to consist of a DC motor running at constant speed. The latter depends on the load voltage which in turn depends upon the frequency of the gate triggering signal $\mathrm{iG}$, the values of the capacitor $\mathrm{C}$, and the size of the SR. The cathode of the thyristor is connected to the centre of the SR. The unsaturated reactance of the SR is assumed to be very large; hence the corresponding current will be negligibly small. The details of circuit can be obtained in reference [20].The appropriate triggering pulses are generated based on the speed, sensed by the arm controller.

\section{A. Methodology used for Speed Computation of DC Motor}

In order to achieve a speed of 10,000 rpm the dc motor used is fed by Morgan chopper to the armature circuit, while maintaining the field constant. The resulting equations describe the relation with speed and chopper voltage applied to armature.

EMF equation of separately excited DC machine is given by

$$
E=\frac{P \varnothing \mathrm{ZN}}{60 A}
$$

Where, $\mathrm{P}=$ No. of poles

$$
\begin{aligned}
& \varnothing=\text { Flux } \\
& Z=\text { No. of armature conductor } \\
& \mathrm{N}=\text { Speed in rpm } \\
& \mathrm{A}=\text { No. of parallel paths } \\
& \quad \begin{aligned}
\text { For wave winding } \mathrm{A}=2 \\
\text { For lap winding } \mathrm{A}=\mathrm{P}
\end{aligned} \\
& \mathrm{E}=\text { Voltage in volts }
\end{aligned}
$$

Since field is kept constant. Voltage $\mathrm{E}$ is directly proportional to speed of the Motor N, mathematically represented as

$$
\operatorname{E\alpha N} \quad---2
$$

\section{In equation 2, varied $\mathrm{E}$ will result in variations of $\mathrm{N}$}

The Optical shaft consists of an opaque disc mounted on the shaft of the motor whose speed is to be measured. The disc has got number of fringes around the periphery of the disc. An opto-coupler is used to measure the speed of the motor. The resulting pulses obtained give one to one correspondence with speed. The fig. 4 shows the construction and the pulses obtained $[1,2,19]$.

\section{B. Measurement of Speed Using Optical Shaft}

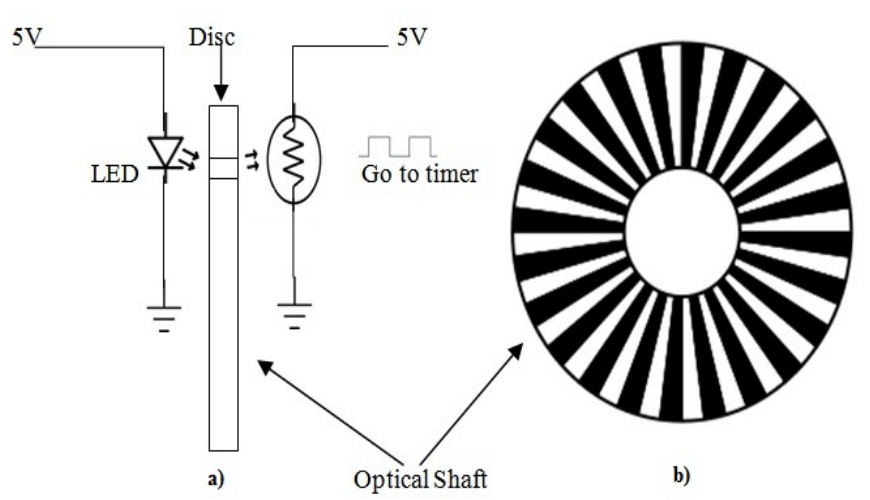

Fig. 4. (a) Measurement of Speed with Disc Fringes, (b) Optical Disc with Shaft of 10000 fringes

The numbers of Pulses obtained are fed to the port of LPC2148 ARM Controller and judicious software computes the speed of the motor by measuring the pulse width count. The count obtained is compared with the look up table to determine the speed. Any variation in speed is corrected by generating a new set of triggering pulses or firing angle to the Morgan chopper using look up table. The flow chart depicts the generation of chopper pulses and speed measurements as shown in fig. 5.

The timer0 is used to count the incoming pulses from the optical shaft. The algorithm mentioned below is used to measure the speed and display the speed on the LCD panel.

If the desired speed of $10000 \mathrm{rpm}$ is not achieved, then a new triggering pulse is generated from timer1.These two operations are continuously polled till the desired speed is obtained. Once the speed is achieved, a delay program of 5 min is used after which the readings of the ADC are recorded to process the count of RBC \& WBC.

\section{LCD Section}

A LCD is used to display the readings of speed and RBC and WBC count. The specification of the LCD used is ADM1602K-NSW-FBS/3.3V [25]. It has two rows of 16 characters in each row. This LCD is interfaced to the ARM through $\mathrm{I} / \mathrm{O}$ ports $\mathrm{P} 1$.

\section{Flow chart for speed determination.}

The LPC2148 ARM Controller is initialized with two ports, one port to sense the speed and the other to send the firing angles $(\alpha)$ to the chopper to get the desired speed of 10,000 RPM. The controller is in a loop until the desired output is reached. The look up table is created and stored in memory for 
future modifications depending upon the need and analysis of the samples where count of the pulse width indicates the speed (N) of the motor in RPM and $\alpha$ indicates the firing angle of the chopper.

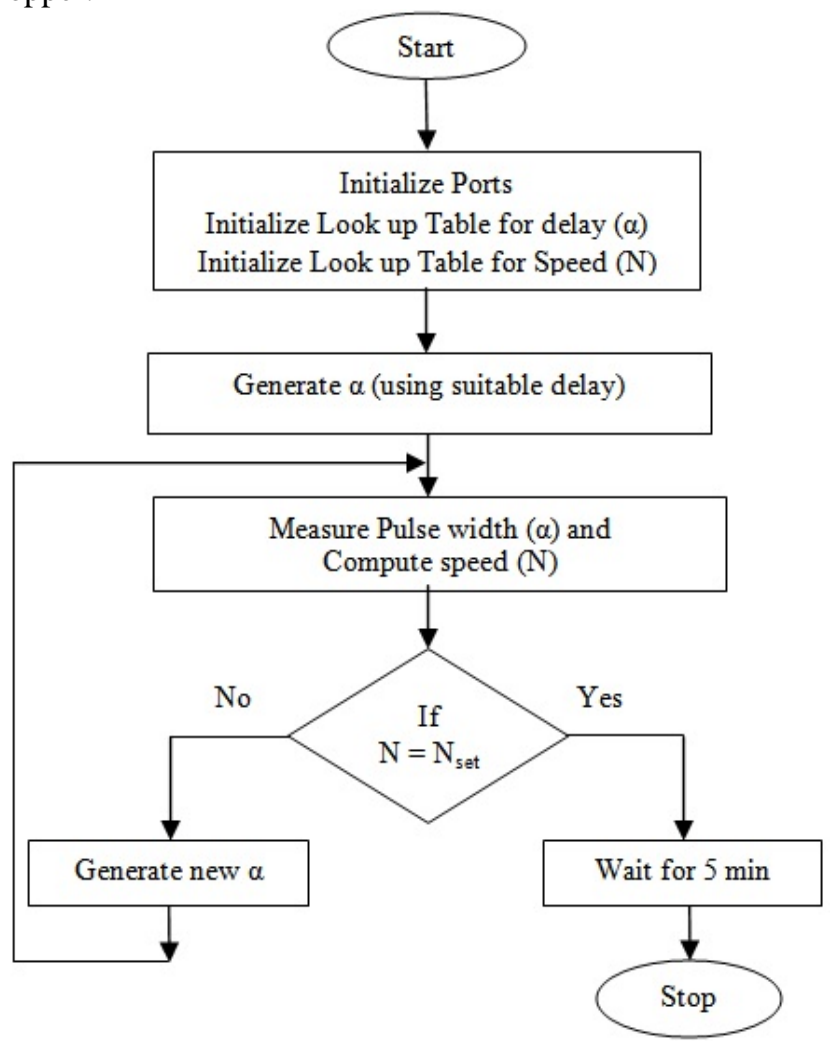

Fig. 5. Flow chart to Determine speed $(\mathrm{N})$ and delay $(\alpha)$.

\section{EXPERIMENTAL SETUP}

The entire set up made for separation and detection of the blood components is as shown in Fig.6.

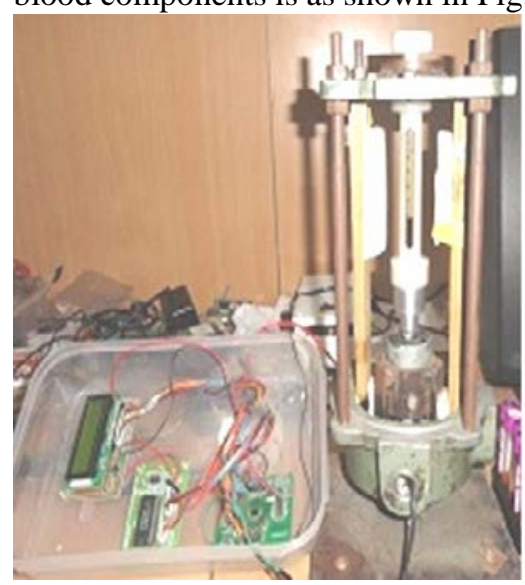

(a)

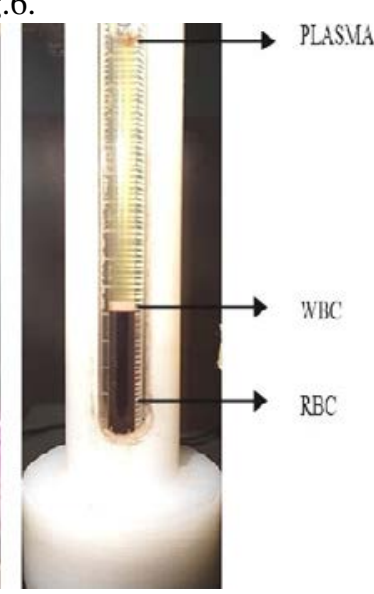

(b)
Fig. 6. a) Experimental Setup b) Sample Column after Separation

A Sample holder containing whole blood, is rotated for a period of 5 minutes with a speed of 10000 RPM to divide blood into individual components. Fig 6(b) shows the resultant sample column with separated components. Since RBC contains some heavy particles such as iron, it settles down at the bottom of the capillary tube (can be seen as red in color) above which the WBC cells get accumulated (can be seen as white in color), then the platelets (can be seen as a yellow circle on top of the WBC). Further, above these three, rests the column of plasma.

Resultant sample column is exposed to a photometric and this system measures the individual components such as RBC and WBC.

TABLE II. RBC Vs A/D READOUT

\begin{tabular}{|c|c|c|}
\hline \multicolumn{3}{|c|}{ RBC Vs A/D readout } \\
\hline $\begin{array}{c}\text { X Axis } \\
\text { (Voltage in } \\
\text { volts) }\end{array}$ & $\begin{array}{c}\text { Y Axis } \\
\text { (RBC count/ul) } \\
\text { (Experimental } \\
\text { reading) }\end{array}$ & $\begin{array}{c}\text { UBM } \\
\text { F } \text {-19 plus } \\
\text { (Auto hematology Analyzer) } \\
\text { RBC count/ul } \\
\text { (Standard reading) }\end{array}$ \\
\hline 1.35 & 3100220 & 3100000 \\
\hline 1.30 & 3300180 & 3300000 \\
\hline 1.25 & 3520100 & 3520000 \\
\hline 1.16 & 3800050 & 3800000 \\
\hline 1.10 & 4000200 & 4000000 \\
\hline 1.09 & 4050200 & 4050000 \\
\hline 1.00 & 4350050 & 4350000 \\
\hline 0.96 & 4500080 & 4500000 \\
\hline 0.93 & 4650080 & 4650000 \\
\hline 0.91 & 4750100 & 4750000 \\
\hline 0.85 & 4999950 & 5000000 \\
\hline 0.79 & 5299920 & 5300000 \\
\hline 0.76 & 5450050 & 5450000 \\
\hline 0.65 & 5899996 & 5900000 \\
\hline 0.58 & 6199996 & 6200000 \\
\hline 0.48 & 6600050 & 6600000 \\
\hline 0.40 & 7000080 & 7000000 \\
\hline 0.38 & 7130080 & 7130000 \\
\hline 0.35 & 7350080 & 7350000 \\
\hline 0.33 & 7500160 & 7500000 \\
\hline 0.30 & 7650020 & 7650000 \\
\hline 0.25 & 7900005 & 7900000 \\
\hline & & \\
\hline
\end{tabular}

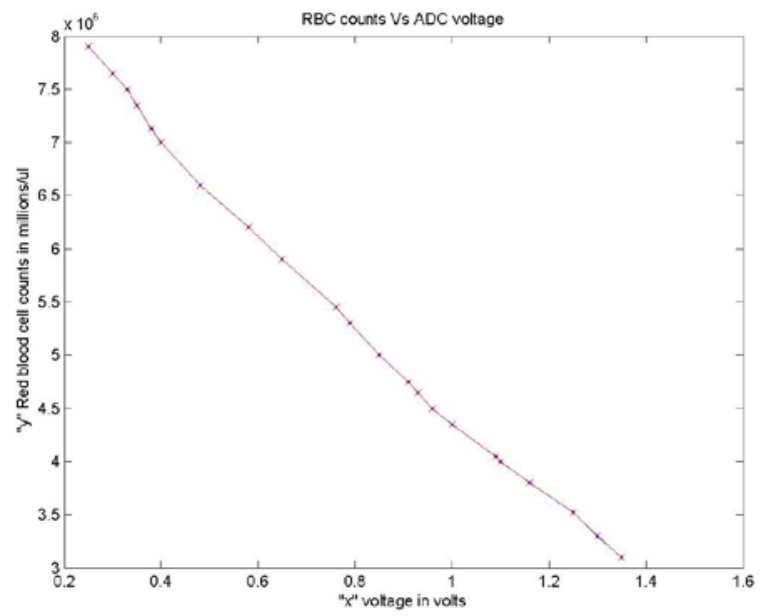

(a) 


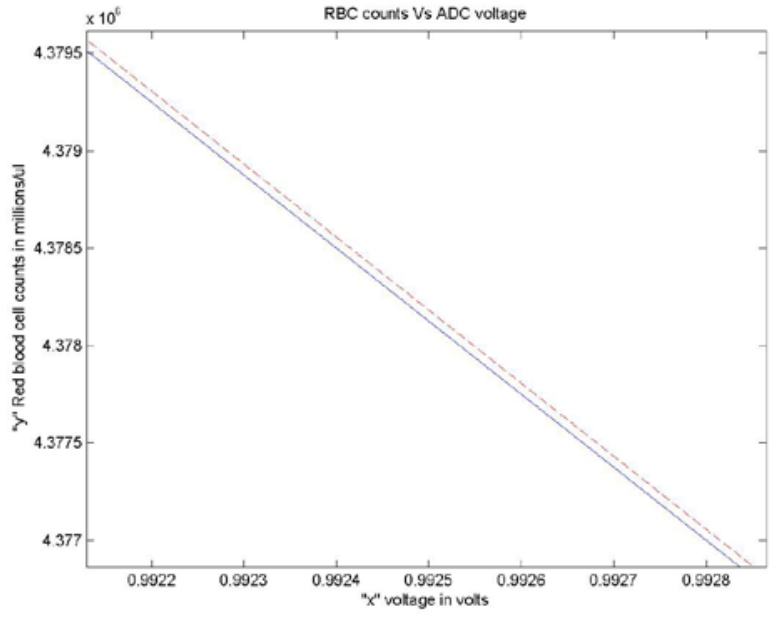

(b)

Fig. 7 (a) RBC Vs Voltage Plot (b) RBC Vs Voltage Plot Expanded

TABLE III. WBC Vs A/D READOUT

\begin{tabular}{|c|c|c|}
\hline \multicolumn{3}{|c|}{ WBC Vs A/D readout } \\
\hline $\begin{array}{c}X \text { Axis } \\
\text { (Voltage in } \\
\text { volts) }\end{array}$ & $\begin{array}{c}Y \text { Axis } \\
\text { (WBC count /ul) } \\
\text { (Experimental } \\
\text { reading) }\end{array}$ & $\begin{array}{c}\text { UBM } \\
\text { FX-19 plus } \\
\text { (Auto hematology Analyzer) } \\
\text { RBC count/ul } \\
\text { (Standard reading) }\end{array}$ \\
\hline 1.48 & 11040 & 11000 \\
\hline 1.50 & 10730 & 10700 \\
\hline 1.52 & 10400 & 10350 \\
\hline 1.56 & 9900 & 9800 \\
\hline 1.58 & 9500 & 9550 \\
\hline 1.62 & 9000 & 9050 \\
\hline 1.68 & 8400 & 8375 \\
\hline 1.69 & 8300 & 8250 \\
\hline 1.76 & 7500 & 7600 \\
\hline 1.81 & 7000 & 7100 \\
\hline 1.85 & 6500 & 6700 \\
\hline 1.97 & 5500 & 5600 \\
\hline 2.01 & 5200 & 5250 \\
\hline 2.03 & 5000 & 5050 \\
\hline 2.05 & 4800 & 4850 \\
\hline 2.09 & 4500 & 4550 \\
\hline 2.12 & 4100 & 4300 \\
\hline 2.14 & 4150 & 4100 \\
\hline 2.17 & 3860 & 3800 \\
\hline
\end{tabular}

RBC \& WBC Vs voltage graph and its enlarged view is shown in the fig 7 and fig 8 . Results are drawn after calibrating photometric system with the standard hematology analyzer (UBM Fx-19).

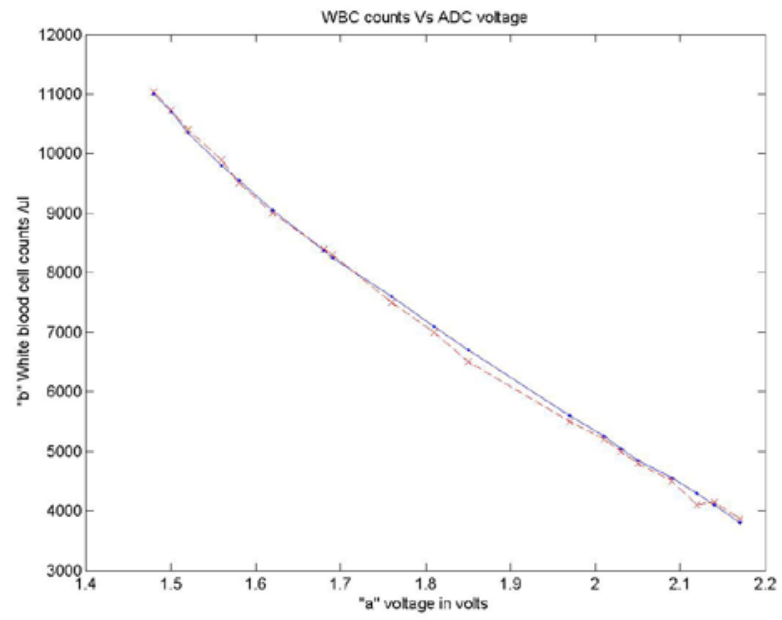

(a)

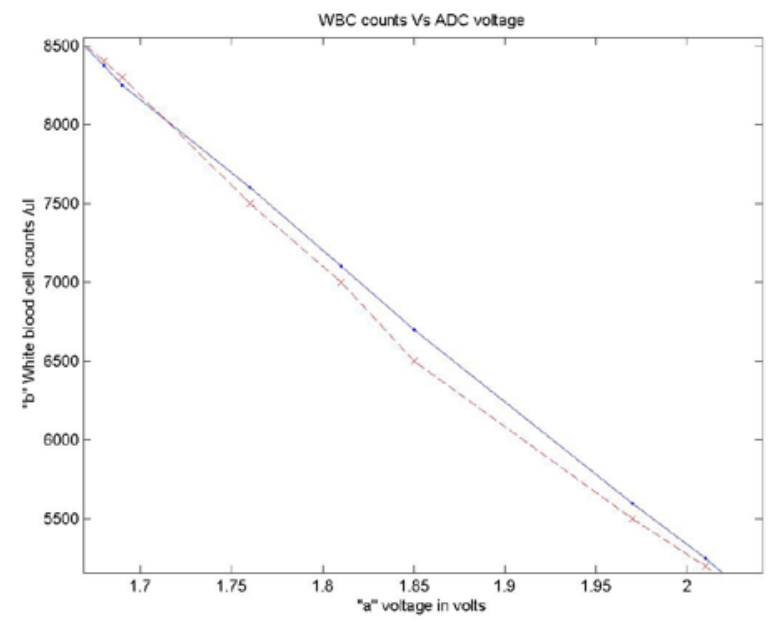

(b)

Fig. 8 (a) WBC Vs Voltage Plot, (b) Expanded Graph of WBC Vs Voltage Plot

The photo detector TSL 257 gives the varying voltage range of $0.25 \mathrm{~V}$ to $1.35 \mathrm{~V}$ and $1.48 \mathrm{~V}$ to $2.17 \mathrm{~V}$ for $\mathrm{RBC}$ and WBC measurements respectively. Further these values are substituted in the calibrated equation to get the RBC and WBC count and then the same is displayed in terms of millions/micro-liter and thousands/micro-liter. Table. II \& III shows the experimental results obtained which are compared with the standard instrument measurements. Table.II shows the RBC count Vs A/D converter read out and Table.III shows the WBC count Vs A/D converter read out measurements (RBC and $\mathrm{WBC}$ ) taken by the standard instrument.

\section{DISCUSSIONS}

The Experimental set up was designed and fabricated. The Expenditure incurred towards the set-up was about Rs.5000=00(Five Thousand Rupees(INR) Only).If the same set-up is used for bulk manufacturing the cost may further decreased and may be easily Commercialized, to meet the needs of the rural Society. 
The experimental set up was tested effectively and the results obtained were critically compared with the standard results obtained from the standard test equipment $\mathrm{Kx}-21$, Transia, Japan (Auto hematology analyzer). The variations obtained were in the order of $\pm 10 \%$. The experimental results obtained are approved by a reputed pathologist. (Certificate Enclosed).

\section{CONCLUSION}

This paper has led to the effective analysis of blood mainly RBC and WBC by using centrifuge method. This method has an advantage of low cost, accurate analysis of blood and can be used in remote areas with the help of battery/DC power. Further, the set up can be made more user-friendly by interfacing this equipment to a PC for data logging which serves as a ready reference for the physicians

\section{ACKNOWLEDGMENT}

We would like to thank Vijayanagar Institute of Medical Sciences (VIMS), Bellary, Karnataka for their support in providing blood samples and using the lab infrastructure in order to calibrate our photometric system.

\section{REFERENCES}

[1] Zeraoulia M, Benbouzid M.E.H,Diallo D (2006), Electric Motor Drive Selection Issues for HEV Propulsion Systems: A comparative Study. IEEE Trans. Vehicular Technology Vol 55, Issues 6.

[2] Safi S.K, Acarnley P P, and Jack A G (1995), Analysis and simulation of the high speed torque performance of dc motor. Vol 142, No 3.

[3] Kersaudy-Kerhoas M, Dhariwal R, Desmulliez M P Y (2007) Recent advances in micro particle continuous separation. IET Nano biotechnology.

[4] Shin S, M.S Park, J.H Jang, Y.H.Ku and J.S Suh (2004) Measurement of red blood cell aggregation by analysis of light transmission in a pressure driven slit flow system. KoreaAustralia Rheology Journal,Vol.16, No.3, pg 129-134.

[5] Dodda Basavana Goud B, Padma Priya K, Nagabhushan Katte (2012) A Review of Recent Advances in Separation and Detection of Whole Blood Components. World Journal of Science and Technology, 2(5):05-09, ISSN: 2231 - 2587

[6] Katsutoshi OOE, Toshio Fukuda, et al (2009) Development of Micro Particles Separation Device with Piezo-Ceramic Vibrator. IEEE.

[7] David W. Inglis(2008) Efficient Microfluidic particle sepration arrays. Department of Physics, Macquaric University Sydney, NSW, 2109, Australia.

[8] Hideaki Tsutsui, Chih-Ming Ho (2009) Cell Separation by noninertial force fields in microfluidic systems. Mechnics research Communications 36,92-103.

[9] Han K H and Frazier A B (2006) Paramagnetic Capture Mode Magnetophoretic Microseparator for Blood Cells. IEEE Proc.Nanobiotechnol. Vol. 153. No 4.
[10] Ae Gyoung Oh, Dong Woo Lee, and Young-Ho Cho. Korea (2010) A Continuous Cell Separator Based On Buoyant Force in Dissimilar Density Fluid Flows. IEEE-Digital Nanolocomotion Center.

[11] Taotao Zhu, Francisco Marrero and Leidong Mao (2010) Continuous Separation of non magnetic particles through negative magnetophoresis inside Ferrofluids. Proceedings of IEEE-international Conference on Nano / Micro Engineered \& Molecular systems. Pg 1006-101.

[12] Kuntaegowdanahalli S S, Bhagat A A S and Papautsky I(2009) Continuous multi particle separation using deterministic focusing in spiral microchannels, Transducers, Pg.2139-2142.

[13] Siyang Zheng, Yu-Chong Tai and Harvey Kasdan (2005) A micro device for separation of Erythrocytes and Leukocytes in human blood. Proceedings of the 2005 IEEE-Engineering in medicine and biology 27th conference, Pg 1024-1027.

[14] Ashkin A, Dziedzic J M, Bjorkholm J E, ET AL (1986) Observation of single-beam gradient force optical trap for dielectric particles. opt.leett. 11,(5),pp.288-290.

[15] Macdonald M P, Spaldind G C, Dholakia K(2003) Microfluidic sorting in an optical lattice. Nature, 426, (6965), pp421-424

[16] Lee C J, Sheen H J, Chu H C, Hsu C J and Wu T H, (2007) The development of a triple-channel separator for particle removal with self-pumping oscillating flow. IOP Publishing Ltd, pp.439-446

[17] Oguz K.Baskurt, Mehmet Uyukle and Herbert J.Meiselman (2010) Time course of Electrical Impedance During Red Blood Cell Aggregation in a Glass Tube:Comparison With Light Transmittance. IEEE Transaction on Biomedical Engineering,Vol.57, No.4

[18] Sunghwan Chang and Young-Ho Cho(2008) Continuous Blood Cell Separation Using a Dielectrophoretic Virtual Pillar array. Proceedings of the 3rd IEEE Int. Conf. on Nano/Micro Engineered and Molecular Systems, Sanya, China

[19] Vincent Del Toro (1985), Electric machines and power systems. PHI private limited, New Delhi.

[20] V.R Moorthi (2009), Power Electronics Devices, Circuits \& Industrial Applications, Oxford University Press, New Delhi.

[21] TSL257- High-sensitivity low-noise light-to-voltage optical converter. IC Data sheets on http://www.ams.com/eng/Products/Light-Sensors/Light-toVoltage-Sensors/TSL257.

[22] LPC2148 Education Board-User's Guide on http://www.embeddedartists.com/products/education/edu_2148. php

[23] User Manual for CP-JR ARM7 USB-LPC2148/EXP on http://www.ett.co.th/download2.html

[24] UM10139 LPC214x User Manual on http://www.nxp.com/documents/user_manual/UM10139.pdf.

[25] User Manual of ADM1602K-NSW-FBS/3.3V LCD on https://www.sparkfun.com/datasheets/LCD/ADM1602K-NSWFBS-3.3v.pdf 